\title{
Calidad de abonos orgánicos empleados en la depresión de Quíbor-Venezuela bajo ambientes protegidos
}

\section{Quality of Organic Fertilizers Used in Greenhouses in Quibor Depression, Venezuela}

\author{
${ }^{\bullet}$ Duilio Torres Rodríguez ${ }^{1}$, Betty J. Mendoza Escalona ${ }^{1}$, Lue M. Marco², Carlos E. Gómez ${ }^{1}$ \\ ${ }^{1}$ Universidad Lisandro Alvarado (UCLA), Departamento de química y suelos, Unidad de investigación en suelos y nutrición \\ mineral de plantas (UISNPM). Cabudare-Lara, Apartado postal 400.Venezuela ${ }^{\triangleright}$ duiliotorres@ucla.edu.ve; \\ bmendoza@ucla.edu.ve; carloseduardo@ucla.edu.ve \\ ${ }^{2}$ Universidad Lisandro Alvarado (UCLA), Departamento de quimica y suelos, Unidad de investigación en análisis instrumental. \\ Cabudare-Lara, Apartado postal 400.Venezuela.mparra@ucla.edu.ve
}

Rec.: 23.05.2016. Acept.: 20.10.2016.

Publicado el 30 de diciembre de 2016

\section{Resumen}

$\mathrm{L}$ a depresión de Quíbor es una de las zonas agrícolas más importante de Venezuela, donde en los últimos años se han introducido sistemas de producción bajo ambientes protegidos, los cuales están acompañados por el uso de abonos orgánicos, no certificados. El objetivo de esta investigación fue evaluar la calidad de los abonos orgánicos empleados en sistemas de producción bajo ambientes protegidos en la depresión de Quíbor, Venezuela. Se realizó un diseño experimental completamente al azar y se evaluaron once (11) abonos orgánicos (cuatro sólidos y siete líquidos) por triplicados. Los abonos sólidos fueron colectados en la parte superior, inferior y media de las pilas, mientras que los líquidos fueron tomados directamente de los tanques de almacenamiento. Para la caracterización de los abonos se empleó el protocolo TMECC, la materia orgánica se determinó por calcinación a $450^{\circ} \mathrm{C}$, el nitrógeno por Kjeldahl y los macronutrientes (potasio y calcio) y micronutrientes (cobre, manganeso, hierro y zinc) en extracto acuoso $1 / 20(\mathrm{v} / \mathrm{v})$ del material y cuantificación por absorción atómica. Los resultados obtenidos muestran que el vermicompost y compost de gallinaza presentaron los valores más altos de macronutrientes, mientras, para los abonos líquidos Macro ${ }^{\circledR}$, fue quien presentó la mayor concentración de nitrógeno, humus ${ }^{1}$ los valores más altos de $\mathrm{Ca}$ y $\mathrm{Mg}$ y Micro® y humus ${ }^{2}$ los mayores contenidos de manganeso, hierro, cobre y zinc fueron encontrados, todos los abonos sólidos presentaron valores bajos de micronutrientes.

Palabras clave: invernaderos, hortalizas, fertilidad, productividad agrícola.

\section{Abstract}

$\mathrm{Q}$ uíbor depression is the main zone for agricultural production in Venezuela, where, during the last years, production systems under coverage have been introduced, together with the use of non-certified organic fertilizers. The objective of this research was to evaluate the quality of organic fertilizers used in production systems in greenhouses in Quibor, Venezuela. A completely randomized experimental design was used and eleven (11) organic fertilizers (four solid and seven liquids) were evaluated for three times. Solid fertilizers were collected in the upper, lower and middle part of the battery, while liquids were taken directly from the storage tanks. For characterizing fertilizers the TMECC protocol was used, organic matter was determined by nitrogen calcination at $450^{\circ} \mathrm{C}$ in Kjeldahl and macronutrients (potassium and calcium) and micronutrients (copper, manganese, iron and zinc), in 1/20 (v/v) material aqueous extract and quantified by atomic absorption. Results show that vermicompost manure and compost had the highest values of macronutrients, whereas for liquid fertilizers

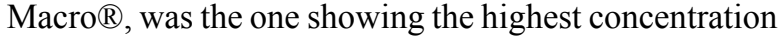
of nitrogen, humus ${ }^{1}$ the highest values of $\mathrm{Ca}$ and $\mathrm{Mg}$, while in Micro ${ }^{\circledR}$ humus $^{2}$ the highest contents of manganese, iron, copper and zinc were found. All solid fertilizers had low levels of micronutrients.

Key words: greenhouse; crop yield, fertility, vegetable. 


\section{Introducción}

L a depresión de Quíbor, ubicada en el centroccidente de Venezuela, constituye una de las zonas más importante para la producción agrícola del país (Mendoza et al., 2013). Se estima que en la misma se produce el $75.80 \%$ de la cebolla, $67.50 \%$ del pimentón y el $25.40 \%$ del tomate, que se consume en Venezuela. Sin embargo, la zona presenta severas limitaciones para la producción agrícola, entre las que se destacan la presencia de suelos salinos, baja estabilidad estructural y susceptibilidad a la erosión. Adicional a las limitaciones naturales, los sistemas agrícolas convencionales, basadas en la aplicación de agroquímicos, el uso excesivo de fertilizantes y la mecanización han conllevado a la degradación y pérdida del potencial productivo de la tierra. Entre los procesos de degradación más notables se encuentran: la compactación del suelo (Reyes, 2014), la salinización (Kimatura et al., 2006) y (Mogollón et al., 2014) la disminución de la materia orgánica (Mendoza et al., 2015) y la pérdida de nutrientes (Delgado-Baquerizo et al., 2013).

Debido a la disminución de la productividad del suelo, los productores se han obligado a la adopción de nuevos sistemas de manejo agronómico como: la rotación de cultivos, la diversificación y la división de los lotes para aumentar el tiempo de descanso de las tierras y la siembra de hortalizas en ambientes protegidos. El cultivo en ambientes protegidos, permite incrementar notablemente la producción debido a que reduce el impacto de las altas temperaturas (De Pascale y Stanghellini, 2011); evapotranspiración (Lorenzo et al., 2010); plagas y enfermedades; sin embargo, uno de los aspectos limitantes para lograr una adecuada producción es la fertilización.

Entre las causas que dificultan el uso de fertilizantes a gran escala esta su alto costo y la escasez de materia prima para su elaboración ( Casanova et al., 1993); no obstante en los últimos años se han desarrollado alternativas como la fertirrigación ( Suniaga et al., 2008) y el uso de abonos producidos a base del compostaje de residuos de cosecha, los cuales poseen un alto contenido de nutrientes, entre estos abonos se pueden citar los vermicompost (Mogollón et al., 2015); la gallinaza (Antonius et al., 2014); los lodos residuales, pulpa de café y abonos foliares a base de sustancias húmicas. La disponibilidad de micronutrientes dependerá de la naturaleza de los materiales empleados en el compostaje como del proceso de descomposición.

El uso de estos abonos conlleva a incrementar el contenido de materia orgánica (Salazar et al., 2009), aumentar la capacidad de intercambio catiónico (Tahboub et al., 2008), mejorar la retención de humedad y favorecer la actividad biológica, creando mejores condiciones físicas, químicas y biológicas que permiten incrementar el rendimiento de los cultivos a mediano y largo plazo, favoreciendo la rentabilidad de los mismos (Beltrán et al., 2009).

A pesar, que en la Depresión de Quíbor, se cuenta con una amplia oferta de fertilizantes orgánicos y biológicos como son: biofertilizantes, compost, vermicompost, bioles entre otros, los mismos no han sido certificados desde el punto de vista nutricional y ambiental, por lo cual, el objetivo de esta investigación fue evaluar la calidad de algunos abonos orgánicos empleados con frecuencia en cultivos protegidos de la depresión de Quíbor, garantizando que los mismos suministren los macro y micronutrientes requeridos para el crecimiento de los cultivos.

\section{Materiales y métodos}

\section{Descripción del área de estudio}

La depresión de Quíbor se encuentra ubicada en la región Centro Occidental de Venezuela y es una de las principales zonas productoras de hortalizas de Venezuela. Los suelos se caracterizan por ser salinos, arcillosos, de baja estabilidad estructural y altamente susceptibles a la erosión (Torres et al., 2014). El clima en la depresión de Quíbor, es seco con un régimen bimodal con un período húmedo principal de mayo-junio y uno secundario, pero mucho menos importante, de octubre-noviembre. La zona se caracteriza por presentar marcado periodos de sequía los cuales son catalogados como sequía extrema (SPI -2 o menos), intensidad severa (SPI entre-1.99 a -1.50 ), moderada (SPI entre -1.49 a -1.00 ) y leve $>$ -1.00 (Olivares, 2016), (Figura 1).

\section{Recolección de abonos y tratamientos de la muestra}

Se realizó un estudio completamente al azar para evaluar la calidad de once (11) abonos orgánicos, siete sólidos y 7 líquidos en la depresión de Quíbor al noroccidente de Venezuela. El muestreo de los abonos sólidos se realizó dentro de las pilas de compostaje, se seleccionaron tres pilas al azar, y se tomaron muestras en la parte superior de la pila, en el centro y la parte inferior de la misma, se homogenizó y se tomó una muestra compuesta de 500 gramos. En caso de los abonos líquidos, se seleccionaron tres tanques de almacenamiento de 200 litros y se tomaron alícuotas de $250 \mathrm{~mL}$, las cuales se trasfirieron a envases de plásticos previamente esterilizados y trasladadas al laboratorio para su análisis. Los abonos sólidos colectados fueron turba canadiense, gallinaza y vermicompost, los cuales se describen a continuación: 


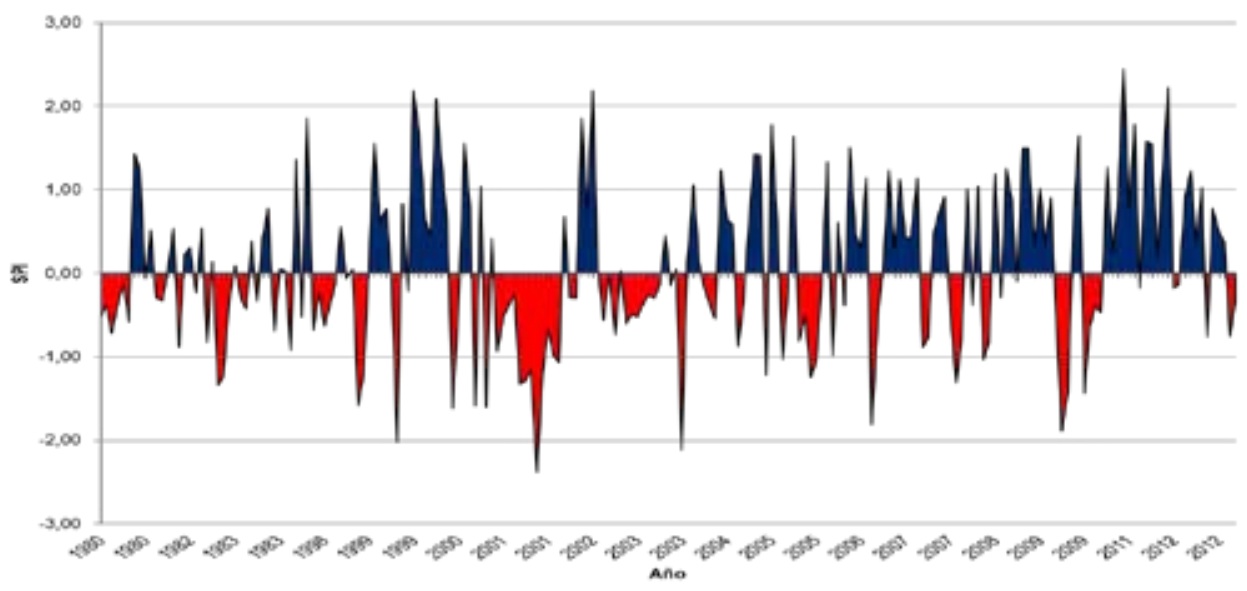

Figura 1. Índice de precipitación estandarizado (SPI) en la estación climatológica Quíbor durante el periodo 1980-2012

Turba canadiense. La turba canadiense fue colectada de tres pilas seleccionadas al azar, dentro de las unidades de producción, las muestras fueron tomadas en la parte superior, inferior y al centro de las pilas, se tomó una muestra compuesta de aproximadamente 500 gramos, almacenada en una bolsa plástica hermética y transportada al laboratorio para su análisis.

Gallinaza. La gallinaza, proviene de la Finca "El Tunal", ubicada a $10 \mathrm{~km}$ de las unidades de producción. La gallinaza fue colectada de tres pilas seleccionadas al azar, dentro de las unidades de producción, las muestras fueron tomadas en la parte superior, inferior y al centro de las pilas, se tomó una muestra compuesta de aproximadamente 500 gramos, la cual fue almacenada en una bolsa plástica hermética, almacenada y transportada al laboratorio para su análisis. El abono fue producido mediante la técnica de aireación por volteos (Gordillo et al., 2011), la formulación más equilibrada para macro y micro nutrimentos y mayor concentración de materia orgánica fue la formulación con $80 \%$ de gallinaza y $20 \%$ de cascarillas de arroz. El proceso de compostaje dura aproximadamente 8 semanas, se realiza el volteo cada dos semanas y se mide la temperatura dos veces por semana, el compost se considera maduro cuando alcanza la temperatura inicial de $45^{\circ} \mathrm{C}$ y alcanza un pH óptimo de 8 a 8.50.

Vermicompost. El vermicompost empleado proviene de la localidad de Caspito municipio Andrés Eloy Blanco del estado Lara, a $1460 \mathrm{msnm}$, precipitación de $1700 \mathrm{~mm}$ año ${ }^{-1}$ y temperatura promedio de $18^{\circ} \mathrm{C}$. El compostaje se realizó usando como materia $10 \%$ de pergamino de café, $10 \%$ de vástago de cambur picado, $0.50 \%$ de urea y $0.80 \%$ de cal dolomítica (Pierre et al., 2009). Para el vermicompostaje, se incorporó el pergamino de café y vástago de cambur a las mezclas, ya que el primero es otro subproducto del procesamiento del café que se desecha en la zona y el segundo un material vegetal no utilizado, generado durante la limpieza de los cafetales. Las mezclas fueron dispuestas en pilas de forma cónica y tapadas completamente con un plástico negro para conservar la humedad y el calor generado en el proceso. Semanalmente se destapaban las pilas y se registraba la temperatura. Posteriormente, se volteaban y mezclaban para luego rehacer la pila y volverla a tapar. La finalización del proceso de compostaje cuándo se alcanzó la temperatura óptima de $45^{\circ} \mathrm{C}$ y $\mathrm{pH}$ entre 8 y 8.50 .

Humus líquidos. Los humus líquidos evaluados fueron dos humus obtenidos a partir de lixiviados de humus de lombriz y un humus derivado de leonardita por un proceso de emulsión y tres biofertilizantes formulados a base de materia orgánica descompuesta. El humus de lombriz y extractos orgánicos lixiviados más la adición de microelementos, denominados Organik ${ }^{\circledR}$ Nitro $^{\circledR}$ Macro $^{\circledR}$ Micro $^{\circledR}$ son comercializados, sin contar con la certificación requerida.

Los lixiviados se preparan mezclando 1 parte de humus y 5 parte de agua, este se deja reposar 48 horas, se agita periódicamente y posteriormente se filtra. Para utilizarlo se diluye 1 parte de concentrado en 4 partes de agua. La leonardita se extrae con hidróxido de potasio, para la obtención del ácido húmico, el cual se mezcló con el lixiviado obtenido de lombricompuesto, en una relación $75 \%$ del lixiviado de lombriz y $25 \%$ de leonardita para la obtención del humus liquido denominado humus ${ }^{3}$. Los abonos Organik ${ }^{\circledR}$ Nitro $^{\circledR}$ Macro $^{\circledR}$ Micro $^{\circledR}$, son productos enriquecidos 
resultantes de mezclar los lixiviados obtenidos del vermicompostaje con otros lixiviados de materiales orgánicos de distinta naturaleza, los cuales fueron enriquecidos con micronutrientes para aumentar el valor nutricional de los mismos.

\section{Variables evaluadas}

Los análisis fueron realizados en el laboratorio de suelos, de la unidad de investigación de suelos y nutrición mineral de plantas (UISNMP) de la Universidad Lisandro Alvarado (UCLA), en la ciudad de Barquisimeto, Venezuela. Una vez, colectados los abonos, se trasladaron al laboratorio y se colocaron aproximadamente 200 gramos sobre papel de filtro, para ser secadas al aire, posteriormente fueron pasadas por un tamiz de $2 \mathrm{~mm}$.

Para la determinación de las características químicas de los abonos sólidos, se preparó un extracto acuoso 1/20 (v/v) del material, obtenido según la Norma TMECC (2002), para ello se mezclaron 20 gramos del abono orgánico con $400 \mathrm{~mL}$ de agua destilada, se agitó durante 20 minutos, se centrifugó a $8000 \mathrm{rpm}$ por 15 minutos y se filtró la suspensión obtenida. El análisis de los elementos solubles en agua $\mathrm{K}, \mathrm{Ca}, \mathrm{Mg}, \mathrm{Fe}, \mathrm{Cu}, \mathrm{Mn}$ y $\mathrm{Zn}$, se realizó en el extracto $1 / 20(\mathrm{v} / \mathrm{v})$ y se cuantificó por espectrofotometría de absorción atómica, En el caso de los abonos líquidos la medición se realizó directamente por determinación de espectrometría de absorción atómica.

La determinación del contenido en materia orgánica total $(\mathrm{MO})$ se realizó siguiendo la metodología TMECC (2002), la cual consistió en secar las muestras a $105^{\circ} \mathrm{C}$ y posteriormente se calcinó a $450^{\circ} \mathrm{C}$, siendo el residuo de la ignición el contenido en cenizas. Como materia orgánica total (MO) se tomó la pérdida de masa por ignición. Ambos parámetros se expresaron como porcentaje de la muestra seca. Para la determinación de la materia orgánica en los abonos liquidos, se tomó una alícuota de $5 \mathrm{~mL}$ y determinó la materia orgánica por el método de Walkley y Black (1934), que consiste en la oxidación con dicromato de potasio $0.05 \mathrm{~N}$ y cuantificación por espectrofotometría a una longitud de onda de $650 \mathrm{~nm}$.

La determinación de nitrógeno total se realizó con el método de Kjeldahl (Sadzawka et al., 2005). El cual consiste en tres fases fundamentales: digestión de la muestra con ácido sulfúrico para la oxidación de la materia orgánica, destilación con hidróxido de sodio y valoración para determinar el amoniaco desprendido, para la determinación del nitrógeno en los humus liquidos, se tomó una alícuota de $5 \mathrm{~mL}$ y se aplicó el mismo procedimiento analítico que el empleado en las muestras de abonos sólidos.

\section{Análisis de los datos}

Se realizó un análisis de varianza (ANOVA) para estimar diferencias entre los tratamientos evaluados; en aquellas variables que presentaron efectos significativos, se realizaron pruebas de comparación de medias de Tukey $(\mathrm{p}<0.05)$. El análisis estadístico fue realizado usando (Infostat, 2008).

\section{Resultados y discusión}

I os resultados encontrados, muestran que existen diferencias significativas $(\mathrm{p}<0.05)$ al comparar la concentración de macronutrientes en los abonos evaluados (Cuadro 1).

Cuadro 1. Comparación (diferencias estadísticas) entre contenidos de materia orgánica y macronutrientes solubles en muestras de abonos orgánicos provenientes de Quíbor estado Lara

\begin{tabular}{lcccccc}
\hline & MO & N & P & Ca & Mg & K \\
\hline Abonos sólidos & $*$ & $*$ & $*$ & $*$ & $*$ & $*$ \\
Abonos líquidos & $*$ & $*$ & $*$ & $*$ & $*$ & $*$ \\
Tipo de abono & $*$ & $*$ & $*$ & $*$ & $*$ & $*$ \\
\hline
\end{tabular}

* indica diferencia significativa según la prueba de Tukey $(\mathrm{p}<0.05)$

\section{Contenido de materia orgánica de abonos sólidos evaluados}

El contenido de materia orgánica fue significativamente superior $(\mathrm{p}<0.05)$ en las muestras sólidas con un promedio de $33.27 \%$ en comparación a las líquidas cuyo promedio fue de $1.87 \%$. En los abonos orgánicos sólidos, las turbas y el vermicompost, presentaron valores de 38.38; 37.31 y $39.63 \%$ respectivamente, los cuales fueron significativamente $(\mathrm{p}<0.05)$ más altos en comparación al compost de gallinaza, donde se encontró un valor de 29.88\% (Figura 2).

Todos los abonos orgánicos, evaluados presentaron un contenido de materia orgánica, superior al $20 \%$, lo cual es más alto que los valores reportados por Angulo et al. (2014) quienes encontraron que el porcentaje de materia orgánica no fue mayor al $20 \%$ al evaluar más de 100 abonos orgánicos, así mismo los valores de materia orgánica encontrados en la presente investigación están por encima de los valores encontrados por Moreno et al. (2014), quienes encontraron valores que oscilaron entre $8.61-24.40 \%$ al evaluar materiales orgánicos de distinta naturaleza.

Pérez et al. (2008) señalan que el contenido de materia orgánica, va depender de la naturaleza del 


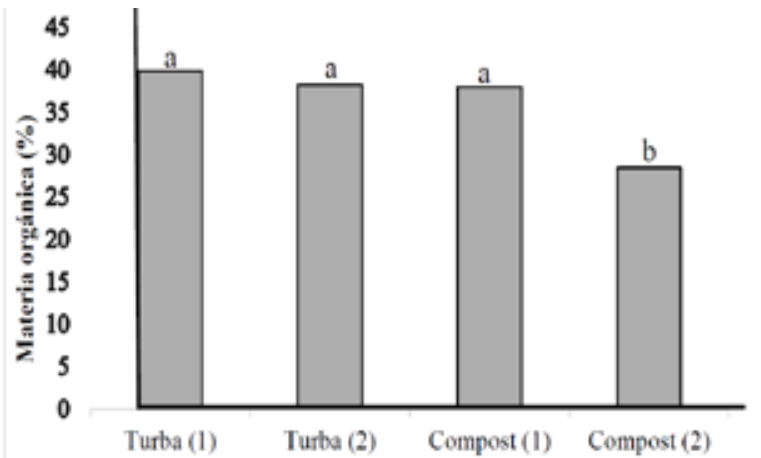

Figura 2. Contenido de materia orgánica de muestras de abonos orgánicos sólidos: Turba ${ }^{1}$ proveniente del sector Rincón de la Guardia; Turba ${ }^{2}$ del sector Campo Lindo; compost ${ }^{1}$ Vermicompost procedente de Sanare; Compost ${ }^{2}$ gallinaza compostada, Hacienda el Tunal

material, del proceso de compostaje y del manejo agronómico del cultivo, estos autores, encontraron valores de materia orgánica más elevados en estiércol de animales en comparación a residuos vegetales. En tal sentido, Moral et al. (2005), Pérez et al. (2008) y Hoyos et al. (2010), encontraron valores de 39.68, 52.20 y 58.60 al evaluar el contenido de materia orgánica en gallinaza, mientras que Contreras et al. (2014), Mogollón et al. (2015) y Muñoz et al. (2015) reportaron valores de $8.85,24.68$ y $27.65 \%$ en vermicompost a base de pulpa de café y otros residuos orgánicos.

El manejo agronómico también pude influir sobre el contenido de materia orgánica de vermicompost a base de residuos de café. Pérez et al. (2008), encontraron valores de $89.30 \%$ en cafetales bajo manejo orgánico y de $20.40 \%$ en cafetales bajo manejo convencional, la gran mayoría de las fincas ubicadas en sanare Venezuela, de donde proviene el vermicompost evaluado, se manejan bajo sistemas de producción convencional. En el caso de las turbas los valores encontrados, estuvieron por debajo de los reportados por Rodríguez et al. (2010) quienes reportaron contenidos de materia orgánica de 79.23 y $58.25 \%$ respectivamente.

\section{Contenido de materia orgánica de abonos líquidos evaluados}

En las muestras líquidas el porcentaje de materia orgánica más alto correspondió al abono foliar Macro con $7.52 \%$, seguido del humus ${ }^{2}$ con $2.40 \%$ cuyos valores fueron estadísticamente superiores $(p<0.05)$ al resto de los abonos evaluados, quienes presentaron valores inferiores al $1 \%$ (Figura 3 ).

El contenido de materia orgánica fue significativamente menor en los abonos líquidos (humus líquido) al compararlo con los abonos sólidos (compost $\mathrm{y}$ vermicompost), los abonos solidos presentaron en promedio $36.12 \%$ de materia orgánica, mientras que en

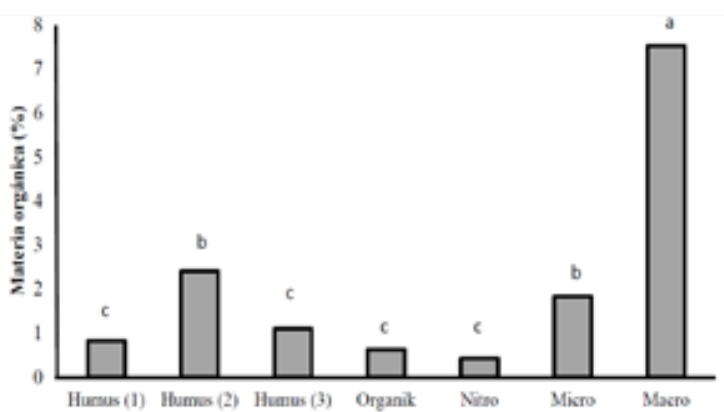

Figura 3. Contenido de materia orgánica en muestras de abonos orgánicos líquidos provenientes del sector Campo Lindo y Rincón de la Guardia. Humus ${ }^{1}$ y $^{2}$ del sector Campo Lindo. Humus ${ }^{3}$ proviene del sector Rincón de la Guardia

los abonos líquidos el contenido de materia orgánica fue de $2.10 \%$. Este comportamiento fue similar al reportado por (Saldaña et al., 2014), quienes encontraron contenidos de materia orgánica más bajos en los humus líquidos al compararlo con abonos orgánicos sólidos. Sin embargo, Ortega y Fernández (2007) y Lazo et al. (2014) encontraron valores de materia orgánica superior al $16 \%$ al evaluar diferentes humus líquidos, estos autores señalan que los humus líquidos en suspensión alcalina a base de leonardita, contienen mayor contenido de materia orgánica, dado que durante el proceso de extracción de la leonardita se producen ácidos húmicos y fúlvicos que al mezclarlo con los te de compost o lixiviados del proceso de compostaje, enriquecen esta mezcla al incrementar el contenido de carbono a través de la fertirrigación, incrementando así la fertilidad del suelo (Rivero et al., 2004 y Rosales et al., 2015).

\section{Contenido de macronutrientes en muestras de abonos orgánicos sólidos y líquidos}

Los abonos sólidos que presentaron el mayor contenido de nutrientes fueron el compost y vermicompost, quienes presentaron valores significativamente más altos de nutrientes $(\mathrm{p}<0.05)$ al compararlos con el contenido de nutrientes de las turbas (Cuadro 2). El mayor contenido de macronutrientes en el compost y vermicompost, se debe a la naturaleza del material compostado, Liang et al. (2003), encontraron que los abonos orgánicos elaborados con gallinaza y pulpa de café poseen alto contenido de nutrientes, Raviv et al. (2004), considera a la gallinaza como un sustrato de alta calidad nutricional, así mismo Contreras et al. (2014) han reportado valores altos de P (0.28\%), y K $(2.5 \%)$ en vermiompost elaborado a base de residuos de café, Díaz et al. (1993) atribuyen el incremento del contenido de nutrientes en los compost de residuos de café a la mineralización de materia orgánica lábil.

El incremento del contenido de nutrientes en los vermicompost es consistente con los resultados 
Cuadro 2. Macronutrientes solubles en muestras de abonos orgánicos sólidos provenientes de Quíbor estado Lara

\begin{tabular}{lcccc}
\hline $\begin{array}{c}\text { Abonos } \\
\text { sólidos }\end{array}$ & $\begin{array}{c}\mathbf{N} \\
(\mathbf{\%})\end{array}$ & $\begin{array}{c}\mathbf{C a} \\
\left(\mathbf{m g ~ L} \mathbf{-}^{-1}\right)\end{array}$ & $\begin{array}{c}\mathbf{M g} \\
\left(\mathbf{m g ~ L} \mathbf{L}^{-1}\right)\end{array}$ & $\begin{array}{c}\mathbf{K} \\
\left(\mathbf{m g ~ L} \mathbf{~ L}^{-1}\right)\end{array}$ \\
\hline Turba $^{1}$ & $0.53 \mathrm{c}$ & $15.84 \mathrm{~b}$ & $8.95 \mathrm{~b}$ & $17.23 \mathrm{~b}$ \\
Turba $^{2}$ & $0.47 \mathrm{c}$ & $15.35 \mathrm{~b}$ & $15.09 \mathrm{ab}$ & $17.57 \mathrm{~b}$ \\
Compost $^{1}$ & $2.65 \mathrm{a}$ & $31.67 \mathrm{a}$ & $23.85 \mathrm{a}$ & $438.33 \mathrm{a}$ \\
Compost $^{2}$ & $0.95 \mathrm{~b}$ & $19.43 \mathrm{ab}$ & $7.85 \mathrm{~b}$ & $378.33 \mathrm{a}$ \\
\hline
\end{tabular}

Turba $^{1}$ proveniente del sector Rincón de la Guardia; Turba ${ }^{2}$ del

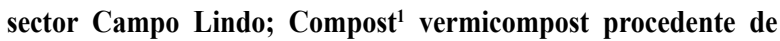

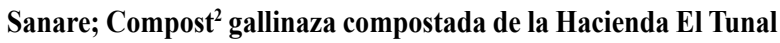

encontrados por (Haritha et al., 2009) quienes reportaron valores más altos de macronutrientes en el vermicompost en comparación a los compost de residuos orgánicos, lo cual es probablemente explicado por la actividad de las lombrices y por la fase termófila. Estos autores sugieren que la descomposición de los residuos orgánicos ingeridos por las lombrices es catalizada por las enzimas secretadas por las mismas y que durante los procesos bioquímicos dentro del proceso de compostaje se liberan además metabolitos secundarios que enriquecen el vermicompost, aumentando su valor nutricional del mismo (Patnaik y Reddy, 2010).

En general, los contenidos de $\mathrm{N}$ fueron similares a los reportados por Giganchi et al. (2003) y Castro et al. (2009), quienes encontraron valores de nitrógeno que oscilaron en el rango de 0.30 a $2 \%$. Todas las muestras a excepción de la Turba $^{2}$, superaron el valor de $0.50 \%$ establecido como referencia por el Instituto Nacional de Normalización Chileno (Angulo et al., 2014).

Valores superiores al $2 \%$ se considera según etiqueta ecológica europea como el límite máximo de $\mathrm{N}$ para un abono orgánico (Castro et al., 2009). No obstante, algunos estudios han reportado concentraciones de $\mathrm{N}$ superiores al 3\% (Contreras et al., 2006; Duran y Henríquez, 2007), asociando estas altas concentraciones a la naturaleza del material seleccionado y a las condiciones ambientales ocurridas durante el proceso de compostaje y vermicompostaje.

El contenido de calcio y magnesio soluble fue bajo en todos los abonos sólidos evaluados al presentar valores por debajo de los rangos de 40-120 $\mathrm{mg} \mathrm{L}^{-1}$ y $50-120 \mathrm{mg} \mathrm{L}^{-1}$ para el calcio y magnesio, respectivamente, los cuales son considerados como óptimos (Meisl et al., 2012). Contrario a lo reportado en esta investigación, Mahmoud e Ibrahim (2012), encontraron valores de calcio superiores a $100 \mathrm{mg} \mathrm{L}^{-1}$ al evaluar un vermicompost a partir de residuos de arroz, sin embargos los valores de potasio en los dos compost evaluados fueron superiores a los valores reportados por estos autores y superan los rangos establecidos como óptimos para este nutriente el cual oscila entre 120-300 $\mathrm{mg} \mathrm{L}^{-1}$ (Meisl et al., 2012).

Los elevados valores de potasio en los abonos solidos evaluados, son explicados por la alta tasa de mineralización de este elemento hacia formas inorgánicas solubles, lo cual hacen que el mismo sea biodisponible y sea aprovechado por las plantas. Por el contrario, otros elementos como el nitrógeno poseen una baja tasa de mineralización (Barrera et al., 2012), adicionalmente parte del nitrógeno puede ser fijado por los microrganismos, debido a los elevados aportes de materia orgánica, especialmente si esta posee elevada relación de $\mathrm{C} / \mathrm{N}$ (Cerrato et al., 2007), esta característica explica que cuando la adición de compost es exclusivamente para suplir deficiencia de fósforo o potasio, las cantidades son menores que cuando se desean suplir los requerimientos de nitrógeno.

En las muestras líquidas los contenidos de nitrógeno fueron significativamente superiores en $\mathrm{Macro}^{\circledR}$ con $1.41 \%$ seguido de humus ${ }^{2}$ con $1.03 \%$, lo cual se corresponde con los altos contenidos de MO, mientras que Organik ${ }^{\circledR}$ y Micro ${ }^{\circledR}$ tienen los contenidos más bajos con 0.09 y $0.05 \%$ respectivamente. (Cuadro 3 ). Calcio y magnesio fueron significativamente superiores en el humus $^{1}$ con respecto al resto de los abonos evaluados al presentar valores de 4521 y $3799 \mathrm{mg} \mathrm{L}^{-1}$, este abono presentó el segundo valor más alto de potasio $(3130 \mathrm{mg}$ $\mathrm{L}^{-1}$ ), después del humus ${ }^{3}$, quien presentó un valor de $8375 \mathrm{mg} \mathrm{L}^{-1}$. Los contenidos de $\mathrm{N}$ del humus ${ }^{2}$ y Macro ${ }^{\circledR}$ fueron superiores a los reportados por Fernández et al. (2009) y González et al. (2013), en humus líquido y té de vermicompost respectivamente, considerados bajos probablemente debido a que el humus utilizado proviene de una solución del lavado de la pila de compostaje, lo que origina que el residuo esté muy diluido (Duran y Henríquez, 2007).

La concentración de nitrógeno en abonos sólidos fue superior $(\mathrm{p}<0.05)$ a la de los líquidos, al presentar

Cuadro 3. Macronutrientes solubles en muestras de abonos orgánicos líquidos provenientes de Quíbor estado Lara

\begin{tabular}{|c|c|c|c|c|}
\hline $\begin{array}{l}\text { Abonos } \\
\text { líquidos }\end{array}$ & N (\%) & $\begin{array}{c}\mathbf{C a} \\
\left(\mathrm{mg} \mathrm{L}^{-1}\right)\end{array}$ & $\begin{array}{c}\text { Mg } \\
\left(\mathrm{mg} \mathrm{L}^{-1}\right)\end{array}$ & $\begin{array}{c}\mathrm{K} \\
\left(\mathrm{mg} \mathrm{L} \mathbf{L}^{-1}\right)\end{array}$ \\
\hline Humus $^{1}$ & $0.16 \mathrm{~b}$ & $4521 \mathrm{a}$ & $3799.00 \mathrm{a}$ & $3130 \mathrm{~b}$ \\
\hline Humus ${ }^{2}$ & $1.03 \mathrm{a}$ & $186 \mathrm{c}$ & $36.00 \mathrm{c}$ & $1839 \mathrm{c}$ \\
\hline Humus ${ }^{3}$ & $0.08 \mathrm{c}$ & $383 \mathrm{~b}$ & $166.00 \mathrm{~b}$ & $8375 a$ \\
\hline Organik $^{\circledR}$ & $0.05 \mathrm{c}$ & $284 \mathrm{c}$ & $35.00 \mathrm{c}$ & $540 \mathrm{~d}$ \\
\hline Nitro $^{\circledR}$ & $0.09 \mathrm{c}$ & $580 \mathrm{~b}$ & $62.00 \mathrm{c}$ & $2260 \mathrm{c}$ \\
\hline Micro $^{\circledR}$ & $0.05 \mathrm{c}$ & $2 \mathrm{c}$ & $0.39 \mathrm{c}$ & $2050 \mathrm{c}$ \\
\hline Macro $^{\circledR}$ & $1.41 \mathrm{a}$ & $531 \mathrm{~b}$ & $125.00 \mathrm{~b}$ & $2380 \mathrm{c}$ \\
\hline
\end{tabular}

Humus $^{1} \mathrm{y}^{2}$ provienen del sector Campo Lindo. Humus ${ }^{3}$ proviene del sector Rincón de la Guardia 
$1.15 \%$ en comparación a $0.37 \%$ encontrado en los abonos líquidos mientras que calcio, magnesio y potasio muestran un comportamiento contrario al nitrógeno, en tal sentido, Mendoza et al. (2011) reportan rangos de contenidos de $\mathrm{K}$, Ca y Mg soluble de (5938-1165 mg $\left.\mathrm{L}^{-1}\right),\left(42.82-234.60 \mathrm{mg} \mathrm{L}^{-1}\right)$ y $\left(161.60-18.21 \mathrm{mg} \mathrm{L}^{-1}\right)$ respectivamente, en compost y vermicompost, rango dentro de los cuales entran la mayoría de los abonos orgánicos líquidos evaluados en esta investigación, incluso superando el rango para los tres nutrientes como ocurre con el humus (Kimatura et al., 2006).

\section{Contenido de micronutrientes en muestras de abonos orgánicos sólidos y líquidos}

Todos los micronutrientes presentaron diferencias significativas $(\mathrm{p}<0.05)$ dependiendo del tipo de abono empleado, a excepción del cobre (Cuadro 4). Al igual que lo observado para el caso de los macronutrientes, los valores más altos de micronutrientes fueron encontrados en las muestras líquidas (Cuadro 5). Los contenidos de cobre, manganeso y zinc solubles en los abonos sólidos son bajos y están por debajo de los valores óptimos establecidos por Ansorena (2011), los cuales son de $1-4 ; 2-30 ; 10-40$ y $1-10 \mathrm{mg} \mathrm{L}^{-1}$ para el $\mathrm{Cu}, \mathrm{Mn}, \mathrm{Fe}$ y $\mathrm{Zn}$ respectivamente. Los resultados son similares a los reportados por Rivera et al. (2008), quienes encontraron valores bajos de estos micronutrientes en compost de residuos agrícolas, estos autores encontraron que el contenido de micronutrimentos se incrementó cuando se adicionó hidróxido de potasio, por lo que la adición de algún elemento durante el proceso de compostaje será clave para la solubilización de los microelementos. Con respecto a los abonos sólidos no se detectó la presencia de micronutrientes en la mayoría de ellos, solamente se reportaron valores muy bajos de cobre, manganeso, hierro y zinc en el compost de gallinaza (Cuadro 6).

Los resultados obtenidos para los abonos sólidos fueron contrarios a los reportados por Lakshmi et al. (2013) y Krishna et al. (2010), quienes señalan que los vermicompost tienen un contenido más alto de micronutrientes en comparación a los compost de residuos vegetales, esto podría ser atribuido a la naturaleza y composición de los residuos orgánicos

Cuadro 4. Comparación (diferencias estadísticas) entre micronutrientes solubles en muestras de abonos orgánicos provenientes de Quíbor estado Lara

\begin{tabular}{lcccc}
\hline & $\mathbf{C u}$ & $\mathbf{M n}$ & $\mathbf{F e}$ & $\mathbf{Z n}$ \\
\hline Abonos sólidos & $*$ & $*$ & $*$ & $*$ \\
Abonos líquidos & $*$ & $*$ & $*$ & $*$ \\
Tipo de abono & $*$ & $*$ & $*$ & $*$ \\
\hline
\end{tabular}

* indica diferencia significativa según la prueba de Tukey $(\mathrm{p}<0,05)$
Cuadro 5. Micronutrientes solubles en abonos orgánicos sólidos y líquidos provenientes de Quíbor, estado Lara

\begin{tabular}{|c|c|c|c|c|}
\hline & $\begin{array}{c}\mathrm{Cu} \\
\left(\mathrm{mg} \mathrm{L^{-1 }}\right)\end{array}$ & $\begin{array}{c}\mathrm{Mn} \\
\left(\mathrm{mg} \mathrm{L}^{-1}\right) \\
\end{array}$ & $\begin{array}{c}\mathbf{F e} \\
\left(m g L^{1}\right)\end{array}$ & $\begin{array}{c}\mathrm{Zn} \\
\left(\mathrm{mg} \mathrm{L}^{-1}\right) \\
\end{array}$ \\
\hline Abonos Sólidos & $0.38 \mathrm{~b}$ & $0.05 \mathrm{~b}$ & $1.61 \mathrm{~b}$ & $0.07 \mathrm{~b}$ \\
\hline Abonos Líquidos & $127.41 \mathrm{a}$ & $349.95 \mathrm{a}$ & $323.49 \mathrm{a}$ & $39.96 \mathrm{a}$ \\
\hline
\end{tabular}

Medias seguidas por la misma letra no presentan diferencias estadísticas (Tukey, p>0.05).

Cuadro 6. Micronutrientes en abonos orgánicos sólidos provenientes de Quíbor estado Lara

\begin{tabular}{|c|c|c|c|c|}
\hline $\begin{array}{l}\text { Abonos } \\
\text { sólidos }\end{array}$ & $\underset{\left(\mathrm{mg} \mathrm{L}^{-1}\right)}{\mathbf{C u}}$ & $\begin{array}{c}\text { Mn } \\
\left(\mathrm{mg} \mathrm{L}^{-1}\right)\end{array}$ & $\begin{array}{c}\mathbf{F e} \\
\left(\mathrm{mg} \mathrm{L}^{-1}\right)\end{array}$ & $\begin{array}{c}\mathbf{Z n} \\
\left(\mathrm{mg} \mathrm{L}^{-1}\right)\end{array}$ \\
\hline Turba (1) & 0 & 0 & 0 & 0 \\
\hline Turba (2) & 0 & 0 & 0 & 0 \\
\hline Compost ${ }^{1}$ & 0 & 0 & 0 & 0 \\
\hline Compost $^{2}$ & 1.92 & 0.26 & 8.05 & 0.39 \\
\hline
\end{tabular}

Turba $^{1}$ proveniente del sector Rincón de la Guardia. Turba ${ }^{2}$ proveniente del sector Campo Lindo. Compost ${ }^{1}$ vermicompost procedente de Sanare; Compost ${ }^{2}$ gallinaza compostada de la Hacienda EI Tunal

usados para el compostaje. En tal sentido Singh et al. (2012), afirman que durante el vermicompostaje las lombrices, pueden acumular altas concentraciones de metales pesados en forma no tóxica, esto permite que el compost tenga menores contenidos de estos elementos. En relación al contenido de micronutrientes los valores de manganeso, hierro, cobre y zinc fueron significativamente superiores en el abono foliar denominado Micro $^{\circledR}$ con 879.28, 2425.58, 1919.09 y $191.26 \mathrm{mg} \mathrm{L}^{-1}$ para el Cu, Mn, Fe y Zn respectivamente, seguido del humus con $6.85,16,26,261.19$ y $64.28 \mathrm{mg}$ $\mathrm{L}^{-1}$ para el $\mathrm{Cu}, \mathrm{Mn}, \mathrm{Fe}$ y $\mathrm{Zn}$ respectivamente. Los valores más bajos de cobre corresponden al humus líquido ${ }^{1}$ con $0.31 \mathrm{mg} \mathrm{L}^{-1}$; los de manganeso a los abonos: Humus ${ }^{1}$ y Humus ${ }^{3}$ con 0.78 y $0.96 \mathrm{mg} \mathrm{L}^{-1}$; los de hierro a Humus $^{1}$ y Nitro ${ }^{\circledR}$ con 8.79 y $8.44 \mathrm{mg} \mathrm{L}^{-1}$; los de zinc a Humus $^{1}$, Organik ${ }^{\circledR}$ y Humus $^{2}$, con 5.50, 3.54 y 1.54, respectivamente (Cuadro 7).

Para el $\mathrm{Cu}$, solamente Macro presentó valores óptimos (1-4 mg L $\left.{ }^{-1}\right)$, Humus ${ }^{1} \mathrm{y}^{3}$, Organik ${ }^{\circledR}$ y Nitro ${ }^{\circledR}$, estuvieron por debajo del rango mientras que Macro $^{\circledR}$ y Micro ${ }^{\circledR}$ por encima del mismo. En el caso del Mn: Nitro $^{\circledR}$, Macro $^{\circledR}$ y humus ${ }^{2}$, estuvieron dentro del rango óptimo $\left(2-30 \mathrm{mg} \mathrm{L}^{-1}\right)$, humus ${ }^{1} \mathrm{y}^{3}$ y Organik ${ }^{\circledR}$, estuvieron por debajo del valor mínimo, en tanto, micro supero el valor máximo permitido. Para el Fe: humus ${ }^{3}$, Organik ${ }^{\circledR}$ y Macro $^{\circledR}$, estuvieron dentro del rango óptimo (10-40 $\mathrm{mg} \mathrm{L}^{-1}$ ), humus ${ }^{1}$ y Nitro ${ }^{\circledR}$ estuvieron por debajo del valor recomendado y humus ${ }^{2}$ y micro ${ }^{\circledR}$, superaron los valores 
máximos permitidos y con relación al $\mathrm{Zn}$ : $\operatorname{Humus}^{1} \mathrm{y}$ ${ }^{3}$, Organik ${ }^{\circledR}$, Nitro $^{\circledR}$ y Macro ${ }^{\circledR}$, estuvieron en el rango optimo (1-10 $\left.\mathrm{mg} \mathrm{L}^{-1}\right)$, mientras que humus ${ }^{2}$ y micro superaron los valores máximos permitidos.

En el caso de abonos como el micro debe tenerse especial cuidado dado que presento altas concentraciones de todos los micronutrientes evaluados, con el propósito de prevenir reacciones toxicas en las plantas (Binner y Schenk, 2013). Los micronutrientes en altas concentraciones causan efectos tóxicos a las plantas, por ejemplo, el $\mathrm{Cu}$ y $\mathrm{Zn}$ está asociado a rizotoxicidad afectando la elongación de las raíces (Michaud et al., 2008).

En cultivo de frijol (Phaseolus vulgaris) altas concentraciones de $\mathrm{Cu}$ y $\mathrm{Zn}$ afectaron significativamente el establecimiento de las plantas (Hojiboland et al., 2006), otros estudios conducidos por Ashagre et al. (2013), reportaron efectos adversos de estos oligoelementos sobre la germinación del tomate (Solanum lycopersicum) y Libralato et al. (2016), reportaron efectos nocivos del $\mathrm{Fe}$ sobre germinación y crecimiento de plántulas, índice de germinación y biomasa vegetal de Berro (Lepidium sativum), mostaza blanca (Sinapis alba) y Sorgo (Sorghum saccharatum). Dado que entre las principales fuentes de contaminantes están los abonos orgánicos mal compostado o con materias primas altamente contaminantes, debe monitorearse las concentraciones de los mismos para evitar efectos tóxicos sobre las plantas.

\section{Conclusiones}

L os abonos sólidos (vermicompost y gallinaza) y líquidos evaluados, presentaron un adecuado nivel de fertilidad, al presentar valores altos de calcio, potasio y magnesio. En el caso de los abonos sólidos, estuvo asociado a los altos niveles de materia orgánica encontrados, mientras, los abonos líquidos fueron relacionados a los procesos bioquímicos catalizados por la lombriz californiana y a la adición de elementos minerales.

La turba canadiense tuvo un alto porcentaje de materia orgánica, no obstante, su uso como abono es restringido, debido al bajo contenido de nutrientes y solo se recomienda su uso como sustrato.

El contenido de micronutrientes fue superior en los abonos líquidos en comparación a los sólidos, a pesar que el mayor contenido de materia orgánica fue en los abonos sólidos. Los abonos líquidos que presentaron la mejor calidad nutricional fueron Micro y humus líquido ${ }^{2}$ al presentar los valores más altos de zinc, cobre, hierro y manganeso.

\section{Agradecimiento}

Ios autores agradecen al Consejo de Desarrollo Científico y Tecnológico de la UCLA (CDCHT) proyecto registrado bajo el código 017-RAG-2012. Planes de fertilización basados en el uso de bioinsumos y recursos locales en El valle de Quíbor, estado Lara y FONACIT proyecto 2012001029. Validación de planes de fertilización basados en el uso de bioinsumos y recursos locales en el valle de Quíbor, estado Lara, por el aporte económico otorgado para la realización de este trabajo.

\section{Bibliografía}

Angulo, J., Ortega, R., Martínez, M., Molina, M., Torres, A. (2014). Evaluation of solid and liquid soil organic amendments for agronomic use in Chile. Acta Horticulturae (ISHS) 1018: 109-114.

Antonius, G., Turley, E., Hill, R., Snider, J. (2014). Chicken manure enhanced yield and quality of field-grown kale and collard greens. Journal of Environmental Science and Health, Part B: Pesticides, Food Contaminants, and Agricultural Wastes 49(4): 299-304.

Ansorena, J. (2011). El compost de bioresiduos en la legislación española. Residuos 21(122): 66-71.

Ashagre, H., Almaw, D., Feyisa, T. 2013. Effect of copper and zinc on seed germination, phytotoxicity, tolerance and seedling vigor of tomato (Lycopersicon esculentum L. cultivar Roma VF). International Journal of Agricultural Science Research 2(11): 312317 .

Barrera, AF., Álvarez-Herrera, JG., Forero, AF., Salamanca, C., Pinzón, L. (2012). Determinación del Nitrógeno potencialmente mineralizable y la tasa de mineralización de nitrógeno en materiales orgánicos. Temas agrarios, 17(1): 32-43.

Beltrán, F., García, J., Ruiz, F., Fenech, L., Murillo, B., Palacios, A., Troyo, E. (2009). Nutrimental potential of red dolichus, brown dolichus and cowpea for green manure produced under three tillage systems. Trop. Subtrop. Agroecos. 10: 487-495.

Binner, I., Schenk, M. (2013). Manganese in substrate clays - harmful for plants?. J. Plant. Nutr. Soil Sci. 176(5): 809-817.

Casanova, E., Goitia, R., Pereira. P., Comerma, J., Aguilar, C. (1993). Necesidades y Perspectivas Agronómicas de Fertilizantes y Enmiendas en Venezuela. Venesuelos 1(1): 17-23.

Castro, A., Henriquez, C., Bertsch, F. (2009). Capacidad de suministro de $\mathrm{N}, \mathrm{P}$ y K de cuatro abonos orgánicos. 
Agronomía Costarricense, 33(1): 31-43.

Cerrato, ME., Leblanc, HA., Kameko, C. (2007). Potencial de mineralización de nitrógeno de bokashi, compost y lombricompost producidos en la universidad Earth. Tierra Tropical 3(2): 183-197.

Contreras, F., Paolini, J., Rivero, C. (2006). Efecto de la adición de enmiendas orgánicas sobre la cinética de la mineralización del carbono en suelos del municipio Rivas Davila, estado Merida, Venezuela. Revista Facultad de Agronomia UCV 31: 37-52.

Contreras, J., Acevedo, I., Rojas, J., Adams, M. (2014). Caracterización de las propiedades físicas y bioquímicas del vermicompost de pergamino de café y estiercol de bovino. Revista Facultad de agronomía LUZ suplemento 1: 489-501.

De Pascale, S., Stanghellini, C. (2011). High Temperature Control in Mediterranean Greenhouse Productio: the Contraints and the Options. Acta Horticulturae, 893: 103-116.

Delgado-Baquerizo, M., Bowker, M., Gallardo, A., Florentino, A., Torres, D., Wellestein MD. (2013). Decoupling of soil nutrient cycles as function of aridity in global drylands. Nature 502: 672-676.

Díaz, L., Savage, G., Eggerth, L., Golueke, C. (1993). Composting and recycling municipal solid waste. Lewis Publishers. Boca Raton.

Fernández, C., Labrador, H., Llobregat, M., Méndez, L., Bastidas, H., Sien, B. (2009). Efectos del estiércol equino y del humus líquido en el proceso de biodegradación de un suelo contaminado con petróleo. Revista de la Facultad de Ingeniería Universidad Central de Venezuela, 24(2): 47-58.

González, K., Rodríguez, M., Trejo, L., Sánchez, J., García, J. (2013). Propiedades químicas de tés de vermicompost. Rev. Mex. Cienc. Agric. 5: 901-911.

Gordillo, F., Peralta, E., Chávez, E., Contreras, V., Campuzano, A., Ruiz, O. (2011). Producción y evaluación del proceso de compostaje a partir de desechos agroindustriales de Saccharum officinarum (caña de azúcar). Revista investigaciones agricolas 37(2): 140-149.

Haritha, D., Vijayalakshmi, K., Kalpana, J., Shaheen, S., Jyothi, K., Surekha, R. (2009). Comparative assessment in enzyme activities and microbial populations during normal and vermicomposting. J. Environ. Biol. 30(6): 1013-1017.

Hojiboland, R., Niknam, V., Ebrahim-Zadeh, H., Mozafari, A. (2006). Uptake, transport and chelation of $\mathrm{Cu}$ and $\mathrm{Zn}$ at toxic levels in tolerant and sensitive species from North West of Iran. J. Sci., 17: 203-214.

Hoyos, J., Vargas, C., Velasco, R. (2010). Evaluación de compost obtenido de pila movil, emplendo mezclas de gallinaza de jaula con material celulolitico. Revista Facultad e Ciencias Agropecuarias 8(1): 55-60.
Infostat. 2008. Manual del usuario versión (2008). Grupo Infostat. Universidad de Córdoba. Argentina.

Krishna, M., Sreenivasan, N., Prakash, S. (2010). Chemical and biochemical properties of Parthenium and Chormolaena compost. Inter.J. Sci. and Nature. 1(2): 166-171.

Lakshmi, R., Rao, P., Sreelatha, T., Madahvi, M., Padmaja, G., Rao, V., Sireesha, A. (2013). Manurial value of different vermicomposts and conventional composts. Global Advanced Research Journal of Agricultural Science 2(2): 59-64.

Lazo, J., Ascencio, J., Ugarte, J., Yzaguirre, L. (2014). Efecto del humusbol (humato doble de potasio y fósforo) en el crecimiento del maiz en fase vegetativa. Bioagro 26(3): 143-152.

Liang, C., Das, K., Maclendon, R. (2003). The influence of temperature and moisture contents regimes on the aerobic microbial activity of a biosolids composting blend Bioresour Technol 86(2): 131-137.

Libralato, G., Costa, A., Zanella, M., Sabbioni, E., Mičetić, I., Manodori, L., Pigozzo, A., Manenti, S., Groppi, F., Volpi, A. (2016). Phytotoxicity of ionic, micro- and nano-sized iron in three plant species. Ecotoxicol Environ Saf. 123: 81-88.

Lorenzo, P., Sánchez-Guerrero, M., Medrano, E., Alonso. F., García, M. (2010). Manejo del clima en el invernadero Mediterráneo. IFAPA. pp. 39-54.

Mahmoud, E., Ibrahim, M. (2012). Effect of vermicompost and its mixtures with water treatment residuals on soil chemical properties and barley growth. Journal of soil science and plant nutrition, 12(3): 431-440.

Meisl, T., Dubský, M., Srámek, F. Necas, T. (2012). The effect of clay amendment on substrate properties and growth of woody plants. Acta Univ. Agr. et Silv. Mend. Brun. 60(8): 163-170.

Mendoza, D., García, F., Belda, R., Fornes, F., Abad, M. (2011). Compostaje y vermicompostaje de residuos hortícolas: evolución de parámetros físicos y químicos durante el proceso. Consecuencias ambientales. Actas Hort. 59: 22-27.

Mendoza, B., Florentino, A., Hernández-Hernández, R., Aciego J., Torres, D., Vera, E. (2013). Atributos biológicos de dos suelos de Quibor con aplicación de abono orgánico y soluciones salinas. Revista Mexicana de Ciencias Agrícolas, 4(3): 409-421.

Mendoza, B., Vera, E., Chassaigne, A., Gómez, C., Torres, D., Bastidad, Y. (2015). Efecto de posición fisiográfica y profundidad en dos sistemas de labranza sobre atributos de un suelo de Turen. Revista Unellez de Ciencia y Tecnología. 33: 1-12.

Michaud, A., Chappellaz, C., Hinsinger, P. (2008). Copper phytotoxicity affects root elongation and iron nutrition in durum wheat (Triticum turgidum durum L.). Plant Soil 310: 151-165. 
Mogollón, J., Martínez ,A., Rivas, W. (2014). Degradación química de suelos agrícolas en la Península de Paraguaná, Venezuela. Suelos Ecuatoriales. 44(1): 2228.

Mogollón, J., Martinez, A. Torres, D. (2015). Efecto de la aplicación de un vermicompost en las propiedades químicas de un suelo salino-sódico del semiárido venezolano. Acta agronómica 64(4): 315-320.

Moral, R., Moreno, J., Perez, M., Paredes, C. (2005). Characterisation of the organic matter pool in manures, Bioresour. Tecgnol, 96: 153-158.

Moreno, A., García, L., Cano, P., Martínez, V., Márquez, C., Rodríguez, N. (2014). Desarrollo del cultivo de melón (Cucumis melo) con vermicompost bajo condiciones de invernadero. Ecosistemas y Recursos Agropecuarios 1(2): 163-173.

Muñoz, J., Muñoz, J., Montes, C. (2015). Evaluación de abonos orgánicos utilizando como indicadores plantas de lechuga y repollo en Popayan, Cauca. Biotecnología en el Sector Agropecuario y Agroindustrial 13 (1): 73 82.

Olivares, B. (2016). Caracterización de la sequía meteorológica en la red de estaciones agrometeorológicas del Instituto Nacional de Investigaciones Agrícolas (INIA) Venezuela. Trabajo de maestría en Tecnología Ambiental de la Universidad Internacional de Andalucía. 69 p.

Ortega, R., Fernandez, M. (2007). Agronomic evaluation of liquid humus derived from earthworm humic substances. Journal of plant nutrition 30(10-12): 2091-2104.

Patnaik, S., Reddy, V. (2010). Nutrient status of vermicompost of urban green waste processed by three-earthworm species- Eisenia foetida, Eudrilus eugeniae and Perionyx excavatus. Appl. and Environ. Soil Sci. 32(3): 15-19.

Pérez, A., Céspedes, A., Núñez, P. (2008). Caracterización física-química y biológica de enmiendas orgánicas aplicadas en la producción de cultivos en República Dominicana. R.C.Suelo Nutr. Veg. 8(4): 10-29

Pierre, F., Rosell, M., Quiroz, A. (2009). El compostaje de la pulpa del café como alternativa para los caficultores. Revista Inia-Hoy 6: 183-190.

Raviv, M., Medina, S., Krasnovsky, A., Ziadna, H. (2004). Organic matter and nitrogen conservation in manure compost for organic agriculture. Compost Science\& Utilization 12: 6-10.

Reyes, W. (2014). Procesos de compactación en un suelo vertisol bajo cuatro condiciones de manejo en la llanura de Coro, estado Falcón, Venezuela. Bioagro 26(1): 39-48.

Rivero, C., Senesi, N., D’Orazio, V. (2004). Los ácidos húmicos de leonardita sobre las características espectropicas de la materia orgánica de un suelo en la cuenca del lago de Valencia. Agronomia Tropical 54(2): 133-144.

Rodríguez, R., Alcantar, E., Covarrubias, G., Zamora, F., García, P., Ruiz, M., Salcedo, E. (2010). Caracterización física y química de sustratos Agrícolas a partir de bagazo de agave tequilero. Interciencias 35(7): 73-82.

Rosales, L., Segura, M., Cervantes, G., Potisek, M., Orozco, J., Preciado, P. (2015). Influencia de los ácidos fúlvicos sobre la estabilidad de agregados y la raíz de melón en casa sombra. Interciencia 40(5): 317-323.

Rivera, L., Trejo, I., Gómez, F., Ramírez, M., Méndez, J., Cisneros, J. (2008). Micronutrimentos solubles en vermicomposta. Rev. Chapingo Serie Zonas Aridas, 7: 29-35.

Sadzawka, R., Carrasco, M., Grez, R., Mora, M. (2005). Métodos de análisis de compost. Instituto de Investigaciones Agropecuarias, Serie Actas $N^{0} 30$, Santiago, Chile, 142 p.

Salazar, S., Trejo, E., Vázquez, V., López, M., Fortis, H., Zuñiga, T., Amado, A. (2009). Distribución de nitrógeno disponible en suelo abonado con estiércol bovino en maíz forrajero. Terra Latinoamericana 27 : 373-382.

Saldaña, M., Gómez, R., Rivera, M., Álvarez, J., Pat, J., Ortiz, C. (2014). The influence of organic fertilizers on the chemical properties of soil and the production of Alpinia purpurata. Ciencia e investigación agraria, 41(2): 215-224.

Singh, J., Kalamdhad, A.S. (2012). Reduction of heavy metals during composting-A Review. International Journal Environmental Protection, 2(9): 36-43.

Suniaga, J., Rodríguez, A., Ramirez, L., Romero, E., Montilla, E. (2008). Fertilización, mediante fertirriego, durante diferentes etapas del ciclo de cultivo del pepino (Cucumis sativus L.) en condiciones de bosque seco premontamo. Agricultura Andina (15): 56-65.

Tahboub, M., Lindemann, W., Murray, L. (2008). Chemical and physical properties of soils amended whit pecan wood chips. Hort Sci. 43: 891-896.

TMECC. (2002). Test methods for the examination of composting and compost. United States Department of Agriculture, and Composting Council Research and Education Foundation, Holbrook, NY

Torres, D., Florentino, A., Ospina,A., Marco, L., Colmenares, C., Yendis, H. (2014). Concentración de clorofila y disponibilidad de nutrientes en suelos con presencia de costra biológica de suelo (CBS). Suelos ecuatoriales 44(2): 90-95.

Walkley, A., Black, A. (1934). An Examination of Degtjareff Method for Determining Soil Organic Matter and a Proposed Modification of the Chromic Acid Titration Method. Soil Sci. 37: 29-37. 